\title{
CONTRIBUCIONES DE UN NUEVO PROCESO DE EVALUACIÓN PSICOLÓGICA A LAS PSICOTERAPIAS
}

\section{CONTRIBUTIONS OF A NEW PSYCHOLOGICAL ASSESSMENT PROCESS TO PSYCHOTHERAPIES}

\author{
Carmen Moreno-Rosset \\ Departamento de Psicología de la Personalidad, Evaluación y Tratamiento Psicológicos \\ Universidad Nacional de Educación a Distancia (UNED). España \\ ORCID: http://orcid.org/0000-0002-7575-1920

\section{Rosario Antequera Jurado} \\ Departamento de Personalidad, Evaluación y Tratamiento Psicológicos. \\ Universidad de Sevilla. España \\ ORCID: http://orcid.org/0000-0002-9192-0536
}

Cómo referenciar este artículo/How to reference this article:

Moreno-Rosset, C. y Antequera-Jurado, R. (2020). Contribuciones de un nuevo Proceso de Evaluación Psicológica a las Psicoterapias. Revista de Psicoterapia, 31(117), 233-248. https://doi.org/10.33898/ rdp.v31i117.388 


\title{
Resumen
}

El Proceso de Evaluación Psicológica constituye el método con el que esta disciplina de la Psicología garantiza su estatus de ciencia. La evaluación psicológica está presente, en mayor o menor medida, en toda actividad psicológica que se relacione con el estudio y análisis de una persona o grupo con el fin de establecer un diagnóstico, una orientación, una selección o una intervención psicológica. En este trabajo se describe un nuevo modelo de Proceso de Evaluación Psicológica que consideramos que puede ser de especial utilidad en el ámbito de las psicoterapias por su versatilidad y flexibilidad. La evaluación y el tratamiento psicológicos están íntimamente interconectados entre sí, aunque las distintas psicoterapias utilicen diferentes procedimientos para evaluar e intervenir. La nueva estructura del Proceso de Evaluación Psicológica que aquí se presenta se divide en dos bloques. El primero (fases 1 a 3) corresponde al "proceso evaluativo" en el que se analiza la naturaleza del problema, se establecen las hipótesis de evaluación, se realiza la formulación de caso y la propuesta de intervención. El segundo bloque (fases 4 y 5) corresponde al "proceso valorativo", dirigido a la implementación y evaluación del tratamiento, en el que las hipótesis que se establecen están orientadas a la intervención psicológica. Consideramos necesario que los estudiantes y profesionales noveles aprendan las tareas imprescindibles para realizar una correcta evaluación psicológica, lo que permitirá junto con la práctica, experiencia y supervisión profesional, conseguir eficacia, eficiencia y efectividad en la aplicación de las psicoterapias, al garantizar la adecuada evaluación del problema y la valoración de su tratamiento.

Palabras clave: Proceso de evaluación psicológica, Psicoterapias.

\begin{abstract}
The Psychological Assessment Process establishes the method through which this psychological discipline guarantees its scientific status. Psychological assessment is present, to a greater or lesser degree, in all psychological activities related with the study and analysis of an individual or a group, with the aim of establishing a diagnosis, an orientation, a selection or a psychological intervention. In this work, a new model of Psychological Assessment Process is described, which we consider to be of special utility in the field of psychotherapy, due to its versatility and flexibility. The psychological assessment and treatment are closely interrelated with each other, although the different psychotherapies employ different procedures to evaluate and intervene. The new structure of the Psychological Evaluative Process presented in this paper in divided into two blocks. The first block (phases 1 to 3), correspond to the "assessment process", in which the nature of the problem is analysed, the evaluative hypotheses are established, the formulation of the case is performed and the proposal of the intervention established. The second block (phases 4 and 5), correspond to the "valuation process" directed at the implementation and the evaluation of the treatment, in which the established hypotheses are oriented towards the psychological intervention. We deem it necessary that both students and new professionals learn the indispensable tasks to perform a correct psychological assessment, which together with practice, experience and professional supervision, would achieve efficiency and effectiveness in the application of psychotherapies, guaranteeing an adequate assessment of the problem and the valuation of its treatment.
\end{abstract}

Keywords: Psychological Assessment Process, Psychotherapies. 
En las revistas de psicoterapia suele ser más habitual leer sobre la evaluación de los procesos en las terapias que sobre el proceso de evaluación psicológica. Sin embargo, durante la intervención psicoterapéutica se realizan una serie de tareas que forman parte del proceso de evaluación psicológica (como la recogida de información, la determinación del problema, hipótesis sobre las causas que lo mantienen o lo han generado y el cómo ayudar al cliente al cambio) por lo que ambos procesos se encuentran interrelacionados. A veces, la evaluación psicológica está implícita en el proceso psicoterapéutico, especialmente en aquellas psicoterapias en las que las técnicas de evaluación psicológica pueden utilizarse tanto para evaluar como para intervenir como, por ejemplo, en la Técnica de Rejilla (Ramírez y Feixas, 2019) o en las estrategias narrativas (García-Martínez, 2019) entre otras.

Otras veces las psicoterapias describen las dinámicas que se establecen entre terapeuta y paciente desde el primer encuentro hasta la conclusión del tratamiento, detallando en el proceso terapéutico las distintas fases: desde la definición del problema, el acuerdo sobre los objetivos de la terapia, la programación terapéutica y las estrategias de cambio y la conclusión del tratamiento (Nardone y Watzlawick, 2012). Estas fases están en claro paralelismo a las fases propuestas por distintos modelos de Proceso de Evaluación Psicológica.

En otras ocasiones, más que un interés manifiesto en el Proceso de Evaluación Psicológica y su aplicación en las psicoterapias, las investigaciones se centran en la evaluación del propio proceso psicoterapéutico y los factores que inciden en los resultados obtenidos. En este sentido se resalta la importancia de aspectos tales como la alianza terapéutica, el número de sesiones o la calidad del vínculo para que se den cambios en el cliente (Botella, 2008).

No es el objetivo del presente trabajo analizar los modelos o estrategias evaluativas que se utilizan (o no) desde las distintas escuelas o teorías psicoterapéuticas, ni tampoco ofrecer un modelo que sea válido para todas las modalidades de intervención psicoterapéutica. Tan sólo pretendemos proponer un modelo que contribuya a sistematizar las tareas de evaluación e intervención y que ayude a la enseñanza y a la formación de los nuevos profesionales, a que los clientes dispongan de unos parámetros que garanticen que las actuaciones psicológicas siguen principios éticos y científicos, que ayude a los psicólogos a mejorar su práctica profesional y, por último, que facilite la supervisión de la labor terapéutica y la investigación sobre la evaluación de los procesos o de los resultados psicoterapéuticos, tan necesarios dentro de nuestra disciplina.

\section{Antecedentes internacionales y nacionales del Proceso de Evaluación Psico- lógica (PEP)}

Son muchos los autores que han descrito el PEP y, cada uno de ellos resalta unas u otras tareas como más relevantes y las desglosa en más o menos fases que suelen oscilar entre 3 y 9. A nivel internacional, cabe citar a la American Psychological Assessment (APA) con la última publicación en 2013 del Handbook of 
Testing and Assessment in Psychology, donde Sara Maltzman (2013) describe la propuesta de PEP de Weiner (2003) que consta de tres fases entre las que no se incluye la valoración de la intervención psicológica.

Asimismo, en 2003 se publicaron desde la Sociedad Europea de Evaluación Psicológica (European Association of Psychological Assessment (EAPA) las Guías para el Proceso de Evaluación (GAP; Fernández-Ballesteros et al., 2003) en las que se consultó un amplio grupo de evaluadores y se llegó a establecer el proceso de evaluación dividido en cuatro fases: 1 . Análisis del caso, 2. Organización e información de los resultados e información al cliente, 3. Planificación de la intervención y 4. Valoración y seguimiento. En cada fase se listan las tareas a realizar de forma minuciosa, ofreciendo un total de hasta 96 tareas o acciones que debe realizar el evaluador para cumplir con las garantías de calidad del PEP.

En España la primera propuesta se sitúa en 1980 y fue realizada por la profesora Fernández-Ballesteros constituyendo desde ese momento el modelo de mayor influencia en el ámbito de la evaluación psicológica. Su más reciente aportación (Fernández-Ballesteros, 2011) se basa en 7 fases divididas en dos partes. Por un lado, el enfoque descriptivo-predictivo con cuatro fases: $1^{\mathrm{a}}$ ) primera recogida de información, $2^{\mathrm{a}}$ ) formulación de hipótesis y deducción de enunciados verificables, $3^{a}$ ) contrastación: administración de tests y otras técnicas de evaluación y $4^{a}$ ) comunicación de resultados: el informe. Y en el enfoque Interventivo-Valorativo, a las fases anteriores añade tres más: $5^{\mathrm{a}}$ ) Plan de tratamiento y su valoración, $6^{\mathrm{a}}$ ) Tratamiento: diseño y administración y, en su caso, evaluación continua y 7ª) Valoración.

Las propuestas del PEP elaboradas por distintos profesores de Universidades españolas pueden consultarse en Moreno-Rosset (2019). Prácticamente todas ellas coinciden en una fase inicial de recogida de información para identificar el problema, una fase de formulación de hipótesis, para cuya comprobación añaden una fase de recogida de datos, análisis de resultados e interpretación de los mismos. Al final del proceso todos los autores vuelven a coincidir en que después de la intervención psicológica se debe repetir la evaluación para valorar la eficacia de la misma, y algunos añaden una evaluación de seguimiento.

\section{El Proceso de Evaluación Psicológica: una nueva estructura (Moreno-Rosset, 2019)}

A partir del estudio crítico y reflexivo de las estructuras y fases de los diversos PEP publicados, y uniendo conocimientos académicos y aplicados, hemos establecido una nueva estructura y contenidos del PEP con el objetivo de que fuera flexible y útil para los profesionales de la Psicología ya que explica de forma fácil y práctica cómo debe realizarse una correcta evaluación psicológica. Entre sus principales aportaciones, que lo diferencian de las anteriores propuestas, resaltamos una mayor interrelación entre las fases destinadas a la evaluación y al tratamiento psicológicos, y la incorporación de la formulación de caso para completar el espacio entre ambos momentos, aspecto destacado por Caro y Montesano (2016). 


\section{Estructura}

Como hemos comentado, las propuestas anteriores sobre el PEP se han dividido entre 3 y 9 fases. Por nuestra parte, consideramos que tres fases no podían englobar todas las tareas necesarias comprendidas desde evaluar el problema hasta valorar la intervención psicológica. Por el contrario, establecer nueve fases podría significar desmenuzar demasiado las tareas y requerir un excesivo tiempo del que no siempre se dispone en la práctica profesional cotidiana. Decidimos que las labores podrían agruparse en cinco fases nombrándolas con la máxima claridad para que sus rúbricas informasen de las acciones a realizar en cada momento. Nos gustaría destacar que no debe identificarse el número de las fases en el PEP con la cantidad de sesiones de evaluación o de intervención psicológicas, ya que para cada una de las fases del PEP se utilizan, según sea necesario, una o varias sesiones. Nuestra nueva estructura del PEP (Moreno-Rosset, 2019) quedó constituida de la siguiente forma (ver Tabla 1).

Tabla 1. Estructura del Proceso de Evaluación Psicológica (Moreno-Rosset, 2019)

\begin{tabular}{|c|c|c|}
\hline FASES DEL PROCESO & TAREAS A REALIZAR & INSTRUMENTOS A APLICAR \\
\hline $1^{a}$ FASE: ESPECIFICACIÓN DE LA & PRIMERA RECOGIDA DE DATOS & \\
\hline $\begin{array}{l}\text { 1.1. Especificar el motivo de la } \\
\text { demanda }\end{array}$ & $\begin{array}{l}\text { a. Determinar la finalidad de la } \\
\text { consulta (diagnóstico, orientación, } \\
\text { asesoramiento, mediación, } \\
\text { selección, intervención, etc.) } \\
\text { y sobre qué problemas/s o } \\
\text { situaciones se deberá evaluar }\end{array}$ & $\begin{array}{l}\text { Aplicar instrumentos que permitan } \\
\text { obtener una información amplia y } \\
\text { genérica, y consultar otras fuentes de } \\
\text { información: } \\
\text { - Entrevista } \\
\text { - Registros de observación }\end{array}$ \\
\hline $\begin{array}{l}\text { 1.2. Establecer las condiciones } \\
\text { históricas y actuales } \\
\text { potencialmente relevantes }\end{array}$ & $\begin{array}{l}\text { b. Reconstruir la historia de la } \\
\text { persona: } \\
\text { - Recoger información sobre las } \\
\text { condiciones socioambientales } \\
\text { actuales y pasadas que puedan } \\
\text { estar provocando, manteniendo } \\
\text { o controlando el problema } \\
\text { - Realizar una primera } \\
\text { aproximación a las variables } \\
\text { personales, biológicas, sociales, } \\
\text { ambientales, etc. relacionadas } \\
\text { con el problema }\end{array}$ & $\begin{array}{l}\text { - Listados o Inventarios de } \\
\text { conductas } \\
\text { - Escalas de apreciación } \\
\text { - Instrumentos de «screening» o } \\
\text { cribado de problemas } \\
\text { - Datos de archivo } \\
\text { - Informes de otros profesionales } \\
\text { - etc. }\end{array}$ \\
\hline $\begin{array}{l}\text { 1.3. Fijar los objetivos para atender } \\
\text { la demanda }\end{array}$ & $\begin{array}{l}\text { c. Establecer los objetivos derivados } \\
\text { de la demanda: concretar sobre } \\
\text { qué aspectos se va a evaluar y las } \\
\text { metas que guiarán la evaluación }\end{array}$ & \\
\hline
\end{tabular}




\begin{tabular}{|c|c|c|}
\hline FASES DEL PROCESO & TAREAS A REALIZAR & INSTRUMENTOS A APLICAR \\
\hline $\begin{array}{l}2^{\mathrm{a}} \text { FASE: FORMULACIÓN DE } \\
\text { HIPÓTESIS DE EVALUACIÓN } \\
\text { VERIFICABLES }\end{array}$ & ETAPA DE ESTUDIO Y REFLEXIÓN & \\
\hline $\begin{array}{l}\text { 2.1. Formular las hipótesis de } \\
\text { evaluación sobre los problemas y } \\
\text { objetivos de la demanda }\end{array}$ & $\begin{array}{l}\text { a. En función de los problemas } \\
\text { relacionados con la demanda, } \\
\text { plantear hipótesis de evaluación } \\
\text { verificables }\end{array}$ & \\
\hline $\begin{array}{l}\text { 2.2. Definir los problemas a evaluar } \\
\text { para comprobar las hipótesis } \\
\text { 2.3. Seleccionar las técnicas o } \\
\text { estrategias evaluativas }\end{array}$ & $\begin{array}{l}\text { b. Seleccionar para cada hipótesis } \\
\text { las variables a evaluar } \\
\text { c. Elegir los instrumentos de } \\
\text { evaluación más adecuados para } \\
\text { cada una de las variables }\end{array}$ & $\begin{array}{l}\text { Elegir instrumentos específicos } \\
\text { de evaluación de cada una de las } \\
\text { variables seleccionadas. Para ello, es } \\
\text { necesario poseer amplia formación } \\
\text { sobre los motivos de la demanda, las } \\
\text { técnicas de evaluación y, los criterios } \\
\text { diagnósticos }\end{array}$ \\
\hline
\end{tabular}




\section{FASES DEL PROCESO \\ 3a FASE: COMPROBACIÓN DE \\ LAS HIPÓTESIS DE EVALUACIÓN, FORMULACIÓN DE CASO \\ Y COMUNICACIÓN DE LOS \\ RESULTADOS}

3.1. Planificar la aplicación de las técnicas evaluativas

3.2. Aplicar las pruebas

3.3. Corregir, interpretar e integrar los resultados

3.4. Comprobar las hipótesis de evaluación

3.5. Establecer la formulación de caso

3.6. Comunicar los resultados del proceso evaluativo. Informe de evaluación

\section{TAREAS A REALIZAR}

INSTRUMENTOS A APLICAR

\section{SEGUNDA RECOGIDA DE DATOS}

a. En función de las características de la persona a evaluar, los tiempos de aplicación y complejidad de cada instrumento, planificar número de sesiones y orden de administración de las pruebas

b. La aplicación de las pruebas debe Se aplican las pruebas incluir informar y/o formar al cliente seleccionadas en la fase anterior para obtener su consentimiento y promover una actitud activa y participativa

c. La corrección e interpretación de cada una de las pruebas e integración de todos los datos permitirá la realización de las restantes tareas de esta fase

d. Determinar la aceptación o rechazo de cada una de las hipótesis. En caso de no verificarse, se deberá repetir la fase anterior, planteando nuevas hipótesis de evaluación verificables

e. A través de un diagrama de tipo pictórico describir la formulación 0 teoría de caso

f. Si el objetivo es realizar un diagnóstico, se ofrecen los resultados del proceso evaluativo de forma oral y, conviene redactar el Informe de evaluación, por si se solicitase en ese mismo momento o pasado un tiempo tenerlo disponible

g. Si el objetivo es realizar una intervención, se añadirá la planificación del programa, por lo que la fase cuatro deberá anticiparse a la comunicación de resultados para comprobar las hipótesis de evaluación y establecer la formulación de caso 


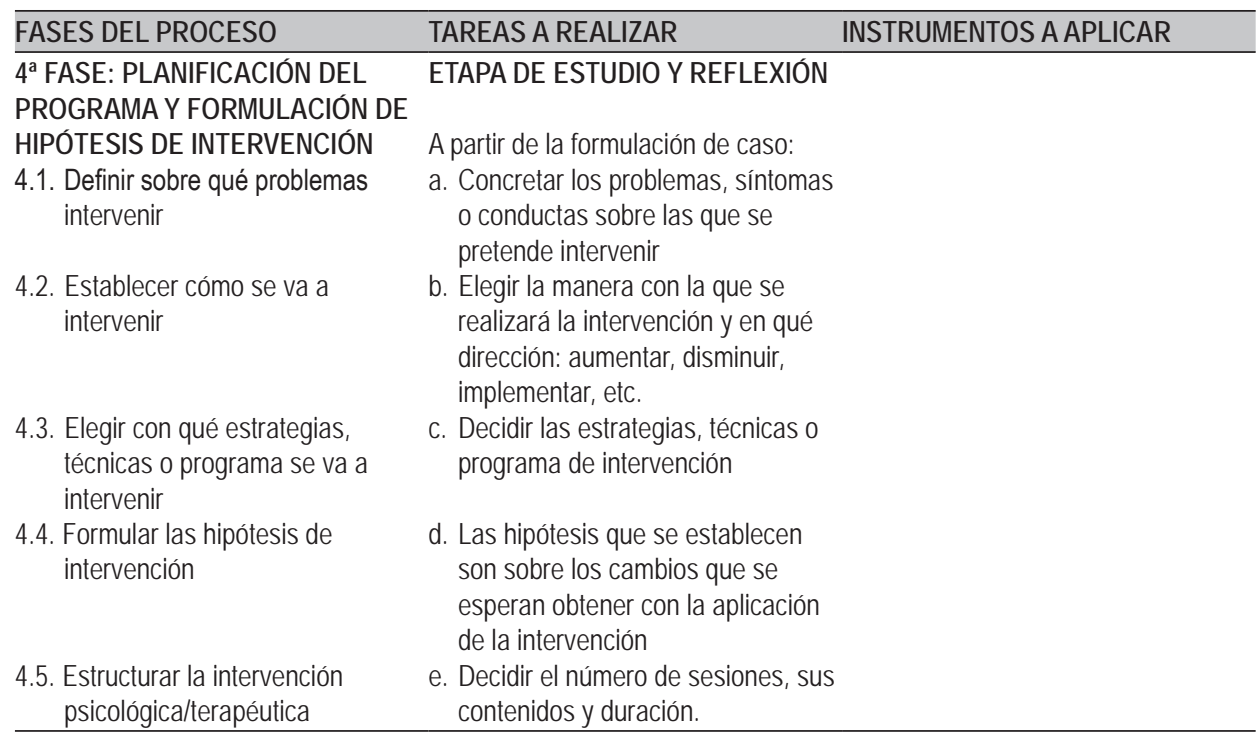

\section{FASE: APLICACIÓN}

Y VALORACIÓN DE LA INTERVENCIÓN PSICOLÓGICA

5.1. Seleccionar los instrumentos de evaluación necesarios para valorar la eficacia de la intervención
5.2. Aplicar las estrategias, técnicas o programa de intervención

5.3. Valorar los resultados y comprobar las hipótesis de intervención

5.4. Comunicar los resultados del proceso valorativo. Informe de intervención

5.5. Preparar para el alta o cierre provisional del caso, si se ha/n previsto seguimiento/s

5.6. Seguimiento/s: valoración del mantenimiento de los objetivos alcanzados

\section{TERCERA RECOGIDA DE DATOS Y}

\section{APLICACIÓN DEL TRATAMIENTO}

a. Comprobar que todos los contenidos de la intervención puedan ser valorados con las pruebas aplicadas en el proceso evaluativo

b. En caso de que algunos no hayan sido evaluados, aplicar los instrumentos de evaluación correspondientes

c. Realizar el tratamiento

d. Realizar una evaluación postratamiento para comprobar el Aplicar todos los instrumentos para la valoración pos-tratamiento, que logro de los objetivos establecidos permitan comparar los resultados con y confirmar las hipótesis de intervención

e. Informar de forma oral y/o escrita de los resultados obtenidos

f. Preparar para el alta

g. En caso de seguimiento/s establecer nuevas fechas

h. En caso de ser necesario, volver a evaluar, para confirmar el mantenimiento de la mejoría

Aplicar los nuevos instrumentos de evaluación necesarios para completar la valoración pre-tratamiento los obtenidos en el pre-tratamiento

Aplicar los instrumentos o las técnicas de evaluación que sean necesarios alcanzada

Las cinco fases del PEP se dividen a su vez en dos bloques: el primero es el "proceso evaluativo" (fases 1 a 3), y el segundo se centra en el "proceso valorativo” (fases 4 y 5), cuyo objetivo es la evaluación de la intervención. Por tanto, 
cuando el objetivo de la evaluación psicológica sea el diagnóstico, orientación, selección, etc., el proceso se llevará a cabo con solamente los tres primeros pasos. Por el contrario, cuando la demanda sea de tratamiento, el PEP se ampliará hasta la quinta fase. Esto no quiere decir que solo se evalúe en las fases iniciales, como tampoco se interviene en solo las últimas. Evaluación y tratamiento se entrelazan $\mathrm{y}$, como venimos resaltando, se trata de una forma ordenada de presentar el PEP, por lo que también hemos diferenciado las fases en las que el terapeuta está en contacto con la persona (fases primera, tercera y quinta) de las que realiza tareas evaluativas o valorativas a solas (fases segunda y cuarta), debiéndose entender que el psicólogo no siempre trabaja en contacto directo con el cliente, y que las tareas de estudio, reflexión, consulta con otros colegas o profesionales, supervisión etc. también forman parte de la labor profesional.

Otra novedad de este nuevo esquema del PEP es la denominación de las hipótesis clasificadas en tan sólo dos tipos; las que se realizan en el "proceso evaluativo" las hemos denominado "hipótesis de evaluación", y las que se postulan en el "proceso valorativo" se llaman "hipótesis de intervención”. De esta forma se distinguen las hipótesis realizadas con fines evaluativos que servirán para ofrecer un diagnóstico o explicación del problema de las de intervención, que se formulan en la comprobación del tratamiento.

El nuevo modelo de PEP añade información sobre cómo optimizar cada fase del proceso cuando el psicólogo ha obtenido suficiente práctica y supervisión para poder agilizar dicho proceso, utilizando menos instrumentos y sesiones de evaluación y obteniendo una mayor eficiencia en el proceso.

\section{$1^{\text {a }}$ FASE: Especificación de la demanda y de los objetivos}

La fase inicial del PEP empieza por especificar el motivo de la demanda, es decir, conocer la finalidad de la consulta para saber cuál es el problema o situación sobre la que se deberá evaluar e intervenir. Además, proponemos establecer las condiciones históricas y actuales potencialmente relevantes para reconstruir la historia pasada y conocer las distintas esferas en las que el problema afecta a la persona y a sus relaciones en la actualidad, siendo conscientes de que durante todo el proceso debemos estar atentos a la continua información que emerge durante las interacciones cliente-terapeuta y que habrá que integrar en el modelo que progresiva y paulatinamente se va construyendo entre ambos. Finalmente, en esta primera fase del PEP conviene fijar los objetivos para atender la demanda que no siempre coincidirán con el problema o problemas planteados por el sujeto, ya que durante la exploración inicial el psicólogo puede haber detectado algún otro conflicto relacionado y que no ha sido hasta ese momento demandado por el paciente. Cuando los objetivos sean varios, conviene centrarse en los más urgentes, siempre de forma consensuada con el paciente al que deberemos oír y respetar lo que considere como sus principales necesidades. Por tanto, a pesar de que una es la persona que pide ayuda y la otra el profesional que se la brinda, las tomas 
de decisiones en cada momento deberán hacerse, desde nuestro punto de vista, conjuntamente, salvo en ámbitos específicos tales como, por ejemplo, el forense o la selección de personal en que las evaluaciones no son directamente solicitadas por el sujeto sino por fiscales, jueces o empresarios respectivamente. En aquellos casos en que el terapeuta detecte algún problema de riesgo para el paciente se debe comenzar la intervención cuanto antes.

Respecto a los instrumentos de evaluación en esta fase, consideramos a la entrevista como prioritaria, recomendando sea abierta y centrada en el entrevistado en la que se utilizan, principalmente, técnicas de respuesta de escucha no directivas (Guillén y Moreno-Rosset, 2019). También pueden utilizarse otros instrumentos de evaluación de amplio espectro, desde técnicas subjetivas (García-Martínez, 2019; Ramírez-Uclés y Feixas, 2019), proyectivas (Gómez de Terreros y Valdés, 2019) o la observación (Caprara y Anguera, 2019). Asimismo, cuando lo requiera el caso, debe entrevistarse a familiares u otras fuentes de información, o contactar con otros profesionales de la salud.

\section{$2^{\text {a }}$ FASE: Formulación de hipótesis de evaluación verificables}

Las tareas para realizar en esta segunda fase son tres. Primero, en base a los problemas y objetivos de la demanda, el terapeuta debe formular hipótesis de evaluación teniendo en cuenta toda la información recogida en la primera fase del PEP y sus conocimientos sobre la problemática del caso. Segundo, debe seleccionar para cada hipótesis las variables a evaluar y, en tercer lugar, pasará a elegir las técnicas o estrategias evaluativas que servirán para comprobarlas. Insistimos en que para todo ello se requiere amplia formación y experiencia profesional.

Respecto a las pruebas para su comprobación, no siempre será necesario aplicar un instrumento evaluativo para cada hipótesis establecida, sino que a veces, puede ser suficiente comprobarla a través de la observación o del informe verbal del propio paciente. Por otro lado, cada evaluador podrá elegir pruebas diferentes en función del modelo psicoterapéutico que aplique. Respecto al número de hipótesis recomendadas, éstas deben ajustarse siempre a los objetivos del caso, por lo que no es necesario realizar una gran cantidad de hipótesis, sino, solo las necesarias para poder poner en marcha, las soluciones al caso cuanto antes. Además, al postular las imprescindibles, se reducirá la cantidad de pruebas de evaluación que, en muchas ocasiones, no hacen más que corroborar la validez convergente entre las mismas. Como resalta Moreno-Rosset (2019), con la entrevista se llega a obtener la mayor parte de información que si seconsidera necesario, puede posteriormente comprobarse a través de otras técnicas de evaluación más específicas (Ramírez-Uclés, 2019).

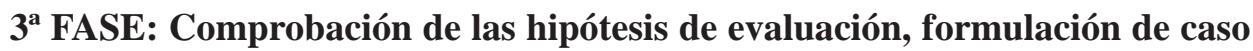 y comunicación de los resultados}

Esta fase cierra el bloque del "proceso evaluativo" con una segunda recogida de datos. A continuación, dividiremos en tres partes la explicación de las tareas 
de esta fase:

\section{a) Comprobación de las hipótesis de evaluación}

La primera tarea consistirá, en planificar la aplicación de las técnicas evaluativas que se habrán seleccionado en la fase anterior. Para ello se deberán tener en cuenta los tiempos necesarios para su administración y calcular las sesiones y materiales necesarios considerando las edades y características de las personas que van a ser evaluadas. Las entrevistas que se apliquen en este momento deberán tener como objetivo la comprobación de hipótesis de evaluación, por lo que se recomienda utilizar "técnicas de entrevista directivas” (Guillén y Moreno-Rosset, 2019).

Esta planificación se podría haber añadido como último paso en la fase anterior, pues es una tarea que se realiza en ausencia de la persona evaluada, sin embargo, la hemos incluido aquí porque se encamina a la verificación de las hipótesis de evaluación planteadas.

El segundo paso en esta fase consistirá en aplicar las pruebas de evaluación psicológica cuya administración conviene consensuar con las personas que van a ser evaluadas, y obtener el correspondiente consentimiento para su aplicación, ofreciéndoles la información necesaria para la correcta recogida de datos a través de su participación activa.

A continuación, se deberán corregir, interpretar e integrar los resultados, que es otro aspecto que el psicólogo realizará en solitario. Corregir e interpretar correctamente una prueba tiene mucha importancia, ya que cualquier dato calculado o entendido de forma errónea puede llevar a conclusiones equívocas. Pero algo todavía más importante es integrar bien los resultados de las técnicas aplicadas desde el principio del PEP para conseguir una correcta interpretación, lo que permitirá comprender mejor la naturaleza del problema, así como precisar la realización de las restantes tareas de esta fase.

La tercera tarea consiste en la comprobación de hipótesis de evaluación para cuya realización conviene concretar "predicciones verificables" sobre los instrumentos de evaluación aplicados. Esto significa que las hipótesis que se establezcan sobre los casos se operativicen, al expresar en las mismas los resultados que se esperan obtener.

Cuando las hipótesis no se verifiquen deberán ser replanteadas, entendiendo que tanto su aceptación como su rechazo es siempre positivo, ya que supone un avance en la comprensión del problema, nos permite reconducir las hipótesis, reelegir las estrategias evaluativas y realizar de nuevo la correspondiente comprobación. Tanto en esta como en el resto de las fases del PEP debemos ser lo suficientemente flexibles para captar y aceptar que nuestros planteamientos pueden ser o no los más certeros, y buscar no solo la comprobación de nuestras hipótesis, sino también de otras hipótesis alternativas. Es este el camino para aproximarnos al conocimiento del otro, en lugar de a la imagen que habíamos creado de él. 


\section{b) Formulación de caso (FC)}

La comprobación de las hipótesis de evaluación y la formulación de caso (FC) se realizan a la par durante la tercera fase del proceso al estar íntimamente conectadas. La FC es un componente esencial en la práctica y entrenamiento en psicoterapia y se establece una vez verificadas las hipótesis y establecidas las variables sobre las que resultará más beneficioso intervenir. Estamos de acuerdo con Ingram (2016) en que antes de proceder a la planificación del tratamiento, es necesario realizar la FC que servirá para conceptualizar los problemas del cliente y orientar el tratamiento oportuno. Montesano y Caro (2016, p. 1) afirman que "las formulaciones que realizamos como psicoterapeutas son el vehículo a través del cual construimos realidades con nuestros clientes" (resaltado por las autoras).

Se trata de una destreza clínica esencial que orienta al terapeuta hacia el tratamiento y cada formulación puede variar en función de la orientación teórica del terapeuta, y puede entenderse como objeto (un informe, análisis funcional, un diagrama, etc.) o como proceso donde la formulación evoluciona dentro de un encadenamiento recursivo de construcción y revisión (Caro y Montesano, 2016). Enlazando con este último significado, consideramos que se podría entender como parte del PEP, y por ello hemos incorporado la FC como una herramienta necesaria y útil para que el evaluador pueda organizar la información obtenida hasta el momento y usarla para explicarle al cliente los problemas y sus interrelaciones, y proponerle las soluciones o psicoterapia pertinente que podrá ser realizada por el propio evaluador o por otro psicoterapeuta. Además, existe suficiente evidencia de que explicar la FC al cliente, facilita la adherencia al tratamiento (Caro, 2017). Se trata de que las personas evaluadas puedan entender sus procesos para convertirse en agentes activos de los cambios que le llevaran a conseguir los objetivos establecidos en la primera fase del PEP.

Respecto a la representación gráfica de la FC, los distintos modelos de psicoterapias han establecido formas distintas de plasmarla, algunas de ellas descritas en el Monográfico sobre "Formulación de Caso en Psicoterapia" (Montesano y Caro, 2016). En nuestro esquema del PEP hemos elegido la representación pictórica propuesta por Godoy y Gavino (2011) por tratarse de diagramas fáciles y sencillos de realizar que dejan bien identificados los problemas. La representación gráfica del problema supone en sí mismo un proceso reflexivo. Determinar cuáles son realmente las variables nucleares del problema y cómo se relacionan entre sí hace que en numerosas ocasiones nos vuelvan a surgir interrogantes, modifiquemos las interrelaciones o la relevancia de las variables consideradas, o tomemos conciencia de que se nos ha "escapado" una pieza importante y necesaria para que podamos formar el "puzzle” completo. Por todo ello, la FC deberá entenderse como dinámica y cambiante con el tiempo debido a los cambios que se pueden producir en la persona evaluada, aparecer como consecuencia del tratamiento, etc., (Muñoz, 2003). 


\section{c) Comunicar los resultados del proceso evaluativo. Informe de evaluación}

Cuando el objetivo de la evaluación psicológica sea solamente de diagnóstico, se exponen los resultados del proceso evaluativo a través de la entrevista de devolución en la que recomendamos técnicas verbales directicas como la "información”, “instrucciones” y “encuadre” (Guillén y Moreno-Rosset, 2019). La FC será útil en esta fase del PEP para explicar los resultados del proceso evaluativo al cliente y de esta forma evitar las etiquetas diagnósticas, si bien como indica Rodríguez-Sutil (2013) la tendencia a clasificar es inevitable en el ser humano y de utilidad para el clínico. También recomendamos redactar por escrito el "informe de evaluación” que se diferencia del de intervención (Calvo y Rodríguez, 2019). Aquí pues terminarían las tres primeras fases del PEP que, como decíamos, configuran el "proceso evaluativo".

Si por el contrario el objetivo de la evaluación psicológica es el tratamiento, la comunicación de resultados se expondrá después de realizar la cuarta fase de "Planificación del programa y formulación de hipótesis de intervención", ya que en la entrevista de devolución, además de transmitir y compartir las conclusiones sobre los problemas del cliente, también se explicará la forma de resolverlos a través de las estrategias, técnicas psicoterapéuticas o programa de intervención que responderá a los objetivos establecidos sobre el caso. Por consiguiente, todas las labores de la cuarta fase del PEP deberán anticiparse a la comunicación de resultados con el fin de exponerlos. Podemos observar aquí un claro ejemplo de la flexibilidad del PEP.

\section{$4^{\text {a }}$ FASE: Planificación del programa y formulación de hipótesis de intervención}

A partir de la FC que proporciona la comprensión del problema del cliente, el terapeuta llega a esta fase en la que fácilmente puede determinar sobre qué problemas, cómo y con qué estrategias, técnicas o programa se va a intervenir. Los terapeutas con experiencia, al plantear la FC realizan estas tareas de manera casi instantánea.

En este momento, además, se deberán formular las hipótesis de intervención sobre los beneficios que se espera obtener del tratamiento, atendiendo las demandas y expectativas (las alcanzables, lógicamente) del paciente. Así pues, en estas hipótesis se relacionan los problemas, síntomas o conductas con los cambios que se esperan obtener a través de las estrategias, técnicas o programas de intervención.

El final de esta fase consistirá en estructurar la intervención psicológica/terapéutica según los modelos teóricos de cada profesional, previendo el número de sesiones, contenidos y duración aproximada. Esto significa que el terapeuta deberá consensuar con el cliente aquellos aspectos que pueden ser prioritarios y ordenarlos, aunque se trabajen en paralelo todos los objetivos terapéuticos.

\section{$5^{\text {a }}$ FASE: Aplicación y valoración de la intervención psicológica}

En esta fase conviene comprobar que las técnicas aplicadas durante el "proceso evaluativo" son suficientes para valorar los contenidos de la intervención. En caso 
de no serlo, se deberán seleccionar los instrumentos de evaluación necesarios y aplicarlos. De esta manera contaremos con todas las pruebas necesarias para la valoración pre-tratamiento. A continuación, se realizará la intervención (que como decíamos antes, ha podido empezar en fases anteriores) por lo que será el momento de aplicar las estrategias o programa de intervención que cada terapeuta, desde del modelo psicoterapéutico en que se fundamente, haya considerado más efectivos para el caso.

Como terapeuta que intenta ayudar al cliente y como miembro de la comunidad científica debemos demostrar la eficacia de nuestras intervenciones. Para ello, una vez terminado el tratamiento procederemos a valorar los resultados y comprobar las hipótesis de intervención, siendo pues necesaria efectuar una evaluación postratamiento que nos permita conocer los logros sobre los objetivos establecidos, confirmar las hipótesis planteadas y comunicar los resultados del proceso valorativo al cliente, tanto de forma oral, como a través del informe de intervención. Y si efectivamente se han cumplido los objetivos propuestos al inicio y son así percibidos por el cliente y el psicólogo, se debe preparar para el alta o si se ha/n previsto seguimiento/s, procederemos a realizar el cierre provisional del caso. En este segundo supuesto, se deberán establecer nuevas fechas para comprobar el mantenimiento de los logros, lo que llevará a la última tarea: la valoración del mantenimiento de los objetivos alcanzados.

\section{Consideraciones finales}

En elámbito de la Evaluación Psicológica, la existencia de modelos que ayudan a estructurar los distintos pasos y tareas que debe dar el profesional, contribuye a que los objetivos se alcancen con una calidad, ética, profesionalidad y validez comprobables. De esta manera, el PEP ayuda a unificar los criterios profesionales a seguir, siendo al mismo tiempo lo suficientemente flexible para permitir su acomodación a las circunstancias concretas del caso que se esté abordando.

El Proceso de Evaluación Psicológica que proponemos, como otros anteriores, abarca desde la primera recogida de información con el cliente hasta la finalización y valoración de la eficacia de la intervención psicológica realizada. Es un reflejo de la estrecha vinculación que existe entre ambos procesos (evaluación e intervención psicológicas), siendo difícil en la práctica profesional delimitar cuándo empieza y termina cada uno de ellos.

Son varias las características del nuevo PEP que consideramos que pueden ser también de utilidad en el ámbito de las psicoterapias. Entre éstas, resaltamos una manera más ágil e intuitiva de relacionar las fases del proceso con las tareas a realizar en cada una de ellas, la sistematización de aquella parte de la labor del terapeuta que se realiza sin la presencia del cliente y que ayuda a la reflexión sobre la propia actuación profesional, la inclusión de la formulación de caso que no sólo sirve para comprender la naturaleza del caso y diseñar las estrategias de intervención sino que también resulta de especial utilidad para la comunicación y consenso 
terapéutico con el cliente y, por último, la división en dos grandes bloques según que el objetivo a conseguir sea puramente evaluativo o de diagnóstico ("proceso evaluativo") o también pretendamos demostrar la eficacia de la intervención psicoterapéutica llevada a cabo ("proceso valorativo").

\section{AGRADECIMIENTOS}

Agradecemos las aportaciones de Isabel Avilés, Mariagiovanna Caprara, Eugenio Carlos Fernández-Ballesteros, Julio Iñiguez, Cristina Jenaro, $\mathrm{M}^{\mathrm{a}}$ Dolores Lanzarote, Javier López-Cepero, Carmen Maganto y Montserrat Peris, en la descripción de casos siguiendo las fases del nuevo Proceso de Evaluación Psicológica, lo que ha permitido observar su utilidad aplicada a diferentes contextos profesionales de la Psicología (Antequera-Jurado, 2019).

\section{Referencias}

Antequera-Jurado, R. (2019). El proceso de evaluación psicológica aplicado en diferentes contextos. En C. Moreno-Rosset e I. M. Ramírez-Uclés (eds.), Evaluación Psicológica. Proceso, Técnicas y Aplicaciones en Áreas y Contextos (pp. 491-617). Sanz y Torres.

Botella, L. (2008). Resultado y proceso en Psicoterapia Cognitivo-Constructivista Integradora. Apuntes de Psicología, 26(2), 229-241.

Calvo, C. y Rodríguez, J. M. (2019). El Informe Psicológico. En C. Moreno-Rosset e I. M. Ramírez-Uclés (eds.), Evaluación Psicológica. Proceso, Técnicas y Aplicaciones en Áreas y Contextos (pp. 116-146). Sanzy Torres.

Caprara, M. y Anguera, M. T. (2019). Observación Sistemática. En C. Moreno-Rosset e I. M. Ramírez-Uclés (eds.), Evaluación Psicológica. Proceso, Técnicas y Aplicaciones en Áreas y Contextos (pp. 249-277). Sanzy Torres.

Caro, C. (2017). Evaluación del modelo de formulación de caso de la Terapia Focalizada en el Emoción [Tesis Doctoral, Universidad Pontificia de Comillas (España)]. Repositorio Comillas. https://repositorio.comillas. edu/xmlui/handle/11531/22682

Caro, C. y Montesano, A. (2016). Una mirada a la formulación de caso en psicoterapia: Introducción conceptual y perspectiva de futuro. Revista de Psicoterapia, 27(104), 5-22. https://doi.org/10.33898/rdp.v27i104.127

Fernández-Ballesteros, R. (2011). El proceso como procedimiento científico y sus variantes. En R. FernándezBallesteros (ed.), Evaluación psicológica. Conceptos, métodos y estudio de casos (pp. 61-100). Pirámide.

Fernández-Ballesteros, R., De Bruyn, E. E. J., Godoy, A., Hornke, L.F., Ter Laak, J., Vizcarro, C., Westhoff, K., Westmeyer H. y Zacagnini, L. J. (2003). Guías para el Proceso de Evaluación (GAP): Una propuesta a discusión. Papeles del Psicólogo, 23 (84), 58-70.

García-Martínez, J. (2019). Técnicas Subjetivas II. Estrategias narrativas. En C. Moreno-Rosset e I. M. RamírezUclés (eds.), Evaluación Psicológica. Proceso, Técnicas y Aplicaciones en Áreas y Contextos (pp. 343375). Sanz y Torres.

Godoy, A. y Gavino, A. (2011). Formulación de casos clínicos. En FOCAD. Formación Continuada a Distancia. Consejo General de Colegios Oficiales de Psicólogos. Decimotercera edición, abril-mayo, 19.

Gómez de Terreros, M. y Valdés, M. (2019). Técnicas Proyectivas. En C. Moreno-Rosset e I. M. Ramírez-Uclés (eds.), Evaluación Psicológica. Proceso, Técnicas y Aplicaciones en Áreas y Contextos (pp. 377-422). Sanz y Torres.

Guillén, F. y Moreno-Rosset, C. (2019). La Entrevista en el Proceso de Evaluación Psicológica. En C. MorenoRosset e I. M. Ramírez-Uclés (eds.), Evaluación Psicológica. Proceso, Técnicas y Aplicaciones en Áreas y Contextos (pp. 185-215). Sanz y Torres.

Ingram, B. L. (2016). Case formulation and treatment planning. En J. C. Norcross, G. R., VanderBos, D. K. Freedheim, y R. Krishnamurthy (eds.), APA handbooks in psychology. APA handbook of clinical psychology: Aplications and methods (pp. 233-249). APA. https://doi.org/10.1037/14861-012 
Maltzman, S. (2013). The assessment process. En K. F. Geisinger, B. A. Bracken, J. F. Carlson, J.-I. C. Hansen, N. R. Kuncel, S. P. Reise, y M. C. Rodriguez (eds.), APA handbooks in psychology. APA handbook of testing and assessment in psychology, Vol. 2. (p.19-34). APA. https://doi.org/10.1037/14048-002

Montesano, A. y Caro, C. (2016). Editorial: Formulación de caso en Psicoterapia. Revista de Psicoterapia, 27(104), 1-3. https://doi.org/10.33898/rdp.v27i104.128

Moreno-Rosset, C. (2019). El Proceso de evaluación psicológica. Un método científico. En C. Moreno-Rosset e I. M. Ramírez-Uclés (Eds.), Evaluación Psicológica. Proceso, Técnicas y Aplicaciones en Áreas y Contextos (pp. 49-113). Sanz y Torres.

Muñoz, M. (2003). Evaluación para el tratamiento II: análisis funcional y formulación clínica. En M. Muñoz, Manual práctico de Evaluación psicológica clínica (pp. 169-220). Sintesis.

Nardone, G. y Watzlawick, P. (2012). La praxis clínica en terapia estratégica: proceso y procedimientos. En G. Nardone y P Watzlawick (eds.), El arte del cambio (pp. 71-112). Herder.

Ramírez-Uclés, I. M. (2019). Técnicas Psicométricas. En C. Moreno-Rosset e I. M. Ramírez-Uclés (eds.), Evaluación Psicológica. Proceso, Técnicas y Aplicaciones en Áreas y Contextos (pp. 217-248). Sanz y Torres.

Ramírez-Uclés, I. M. y Feixas, G. (2019). Técnicas Subjetivas I. Procedimientos estructurados. En C. MorenoRosset e I. M. Ramírez-Uclés (eds.), Evaluación Psicológica. Proceso, Técnicas y Aplicaciones en Áreas y Contextos (pp. 315-341). Sanz y Torres.

Rodríguez-Sutil, C. (2013). El fantasma del Psicodiagnóstico. Clínica Contemporánea, 4(1), 29-44. https://doi. org/10.5093/cc2013a2

Weiner, I. B. (2003). The assessment process. En J. R. Graham y J. A. Naglieri (eds.), Handbook of-psychology: Vol. 10. Assessment psychology (pp. 3-25). Wiley. 